\title{
MAXIMAL CLOSED PREPRIMES IN BANACH ALGEBRAS $\left({ }^{1}\right)$
}

\author{
BY \\ P. CIVIN AND C. C. WHITE
}

Introduction. In this work we endeavor to investigate the possibility of utilizing the notions introduced by D. K. Harrison in his Memoir, Finite and infinite primes for rings and fields [5], in an attempt to provide an apparatus which relates to the closed subalgebras and the closed subrings of a given Banach algebra.

For a ring $R$ with identity 1 , Harrison defines a preprime as a nonvoid set closed under addition and multiplication and not containing -1 . He calls a maximal preprime a prime. A natural distinction is made between the primes which do not contain 1, the finite primes, and the primes which contain 1, the infinite primes.

The fruitfulness of a direct application of the Harrison theory of primes to a Banach algebra appears unlikely. The natural quotient structures which appear in profusion lose their topological character as the items factored out are not closed topologically. Those primes which are closed topologically are shown in $\S 2$ to have a very special form and to be in the case of a complex commutative Banach algebra in one-to-one correspondence with the maximal ideals of the algebra. By contrast the maximal closed preprimes appear in a variety of forms even in the simplest types of commutative Banach algebras. As with primes, the maximal closed preprimes are called infinite or finite according to whether or not they contain 1.

In $\$ 1$ we obtain the elementary properties of maximal closed preprimes and closed primes. $\$ 2$ is devoted to the identification of the closed primes among the maximal closed preprimes and to their description in more classical terms. The finite maximal closed preprimes are examined in $\S 3$, and one sees that these involve closed subrings rather than closed subalgebras of the given Banach algebra. One also sees the automatic introduction of certain special fields other than the traditional reals, complexes and quaternions.

The final section exhibits as much of a classification scheme as possible for the infinite maximal closed preprimes within the framework of function algebras. The study is brought to the point of making contact with the array of measure theoretic technique that is available for the study of function algebras.

Received by the editors January 18, 1968 and, in revised form, June 16, 1969.

( $\left.{ }^{1}\right)$ The authors gratefully acknowledge support of the work under NSF grant no. GP-5789 and grant no. GP-7516.

The authors wish to thank the referee for suggesting shorter versions of some of the proofs. Copyright (C) 1970. American Mathematical Society 
The authors wish to thank D. K. Harrison for his stimulating seminar covering his work on primes and for allowing them access to a preliminary version of [5].

1. Definitions, examples, and basic results. We adopt the following notation. Let $\boldsymbol{Z}, \boldsymbol{R}$, and $\boldsymbol{C}$ denote respectively the integers, the reals, and the complexes. Let $N$ and $\boldsymbol{R}^{+}$denote the nonnegative integers and the nonnegative reals.

We consider only real or complex Banach algebras with identity, which we denote by 1 , and for convenience we take $\|1\|=1$. In particular, the zero algebra is excluded from consideration. We are at times forced to consider real subalgebras of a given complex Banach algebra. In each such instance we shall indicate the special usage, and the term "subalgebra" without any modifier will indicate that the same field is in use as in the original algebra. When no reference is made to the field, it may be taken as either $\boldsymbol{R}$ or $\boldsymbol{C}$, and a result stated without reference to the field is valid over either.

Every prime, and every maximal closed preprime, must contain the element 0 . A straightforward application of Zorn's lemma shows that every preprime is contained in a prime. In order to show that every closed preprime is contained in a maximal closed preprime, we need the following result.

Proposition 1.1. Let $Q$ be a closed preprime of the Banach algebra A. Then for each $x$ in $Q,\|x+1\| \geqq 1$.

Proof. Suppose $\|x+1\|<1$ for some $x$ in $Q$. Then $-1+(x+1)^{n} \rightarrow-1$. But for every $n$, the element $-1+(x+1)^{n}$ is in $Q$. Since $Q$ is closed, we conclude that -1 is in $Q$, contradicting the fact that $Q$ is a preprime.

COROLlaRY 1.2. Every closed preprime in a Banach algebra $A$ is contained in a maximal closed preprime.

EXAMPLE 1.3. The set $\boldsymbol{R}^{+}$is a closed prime, and therefore a maximal closed preprime, in either $\boldsymbol{R}$ or $C$. For a fixed prime integer $p$, the set $Z p$ is a prime in the ring $\boldsymbol{Z}$, and a maximal closed preprime in $\boldsymbol{R}$, but is not a prime in $\boldsymbol{R}$.

Our next result, which is interesting in its own right, is useful in $\S 4$.

Proposition 1.4. Let $Q$ be a closed preprime of the Banach algebra A, and suppose that $\boldsymbol{R}^{+} \subset Q$. Then for each $x \in Q$ there is some $\lambda \in \boldsymbol{R}^{+}$such that $\lambda$ is in the spectrum of $x$.

Proof. Let $x \in Q$. Let $\lambda \in R^{+}$be such that $\lambda>\|x\|$. Then one checks that $(\lambda-x)^{-1} \in Q$. Suppose that $(\alpha-x)^{-1}$ exists in $A$ for each $\alpha \in[0, \lambda]$. We know that $-x^{-1} \notin Q$, for otherwise $-1=x\left(-x^{-1}\right) \in Q$. Since $Q$ is topologically closed, there is a smallest positive real number $\beta$ such that $(\beta-x)^{-1} \in Q$. Let $\mu$ be any positive real number such that $\mu>\max \left(\beta^{-1},\left\|(\beta-x)^{-1}\right\|\right)$. Then $\left(\mu-(\beta-x)^{-1}\right)^{-1} \in Q$. Now $\left(\mu-(\beta-x)^{-1}\right)^{-1}=(\beta-x)(\mu(\beta-x)-1)^{-1} \in Q$, so $(\mu(\beta-x)-1)^{-1} \in(\beta-x)^{-1} Q$ $\subset Q$. Thus $((\mu \beta-1)-\mu x)^{-1} \in Q$, so $\left(\left(\beta-\mu^{-1}\right)-x\right)^{-1} \in Q$. But $\mu>\beta^{-1}$, so $\beta>\beta$ 
$-\mu^{-1}>0$, contradicting the fact that no positive number $\delta<\beta$ is such that $(\delta-x)^{-1} \in Q$.

It is clear that a closed prime is automatically a maximal closed preprime. In the balance of this section we discuss some of the special properties of the closed primes.

Proposition 1.5. Let $P$ be a closed prime in the Banacin algebra A. Then $1 \in P$, i.e. $P$ is infinite.

Proof. We show that finite primes of $A$ are not closed. Let $Q$ be a finite prime of $A$. We show $1 \in Q^{-}$. Let $Q+N=\{q+n: q \in Q, n \in N\}$. Since $Q+N$ properly contains $Q,-1=q+n$, for some $q \in Q, n \in N$ and $n \neq 0$. Thus $-n-1=q \in Q$ and so $n+1=q^{2}+n q \in Q$. By Proposition 2.1 in [5] it follows that every element of the form $(n+1)^{1 / k}, k$ a positive integer, is in $Q$. Thus $1 \in Q^{-}$and $Q$ is not closed.

Proposition 1.6. Let $P$ be a closed prime in the Banach algebra A. Then $\boldsymbol{R}^{+} \subset P$.

Proof. It is enough to show that $\frac{1}{2} \in P$, since $P$ is closed and the set

$$
\left\{m\left(\frac{1}{2}\right)^{n}: m, n \in N\right\}
$$

is dense in $R^{+}$. Suppose that $\frac{1}{2} \notin P$. Consider the set

$$
\left\langle P, \frac{1}{2}\right\rangle=\left\{p_{0}+p_{1}\left(\frac{1}{2}\right)+\cdots+p_{n}\left(\frac{1}{2}\right)^{n}: n \in N, p_{i} \in P, i=0, \ldots, n\right\} .
$$

Now $\left\langle P, \frac{1}{2}\right\rangle$ is closed under addition and multiplication and properly contains $P$. Therefore $-1 \in\left\langle P, \frac{1}{2}\right\rangle$, and so we may write $-1=p_{0}+p_{1}\left(\frac{1}{2}\right)+\cdots+p_{n}\left(\frac{1}{2}\right)^{n}$. Thus $-2^{n}$ is in $P$. But $1 \in P$ by Proposition 1.5 , so

$$
-2^{n}+\sum_{k=1}^{2^{n}-1} 1=-1 \in P .
$$

If $S$ is an arbitrary infinite maximal closed preprime in a Banach algebra $A$, then $S$ need not necessarily contain $\boldsymbol{R}^{+}$, as the following example shows.

EXAmPLE 1.7. Let $X$ be the closed interval $[-5,-1]$ of real numbers, and let $A=C^{R}(X)$, the real Banach algebra, with supremum norm, of all real-valued continuous functions on $X$. Let $S_{0}$ be the preprime consisting of all polynomials in the real variable $x$ with integer coefficients and with constant term a nonnegative integer. It follows from Theorem 4.2 of [7] that $S_{0}$ is a closed subset of $C^{R}(X)$. Therefore, by Corollary 1.2, there is a maximal closed preprime $S$ of $C^{R}(X)$ with $S_{0} \subset S$, and $S$ is infinite. Consider the polynomial $f$ where $f(x)=x$. Clearly $f \in S_{0} \subset S$. We now claim that $\frac{1}{5} \notin S$. For if $\frac{1}{5} \in S$ then $\frac{1}{5} f$ would be in $S$. But $\left\|\frac{1}{5} f+1\right\|=\frac{4}{5}<1$, contradicting Proposition 1.1. Hence $\frac{1}{5} \notin S$.

Definition 1.8. Let $Q$ be any preprime of the Banach algebra $A$. Then $Q$ is called archimedean if for each $x \in Q$ there is an $n \in N$ such that $n-x \in Q$.

Definition 1.9. Let $Q$ be any infinite preprime of the Banach algebra $A$. Then we define $Q=\{x \in Q:-x \in Q\}$, and $Q^{+}=\{x \in Q:-x \notin Q\}$. 
Proposition 1.10. Let $P$ be a closed prime in the commutative Banach algebra A. Then $P$ is archimedean.

Proof. Let $x \in P$. We choose an $n \in N$ with $n>\|x\|$. Clearly $(n-x)^{-1} \in P$ and in fact $(n-x)^{-1} \in P^{+}$since otherwise $-1=x(n-x)^{-1}-n(n-x)^{-1} \in P$. By Proposition 1.6 of [5] it follows that $n-x \in P$.

Proposition 1.11. Let $P$ be a closed prime in the complex commutative Banach algebra $A$. Then for each $x \in \boldsymbol{P}$, ix $\in \boldsymbol{P}$.

Proof. Let $x \in \boldsymbol{P}$ and suppose $i x \notin P$. Then $-1=p_{1}+i x p_{2}$, since otherwise $P+i x P$ is a preprime properly containing $P$. Thus $-1-p_{1}=i x p_{2}$ and

$$
-1=p_{1}^{2}+2 p_{1}+x^{2} p_{2}^{2} \in P .
$$

We have shown that $R^{+} \subset P$ for any closed prime $P$ in a Banach algebra $A$. If $A$ is commutative, then $P$ is also archimedean and is such that $i \boldsymbol{P} \subset \boldsymbol{P}$. It will be shown in $\S 2$ that if $S$ is any maximal closed preprime of the commutative Banach algebra $A$ which possesses the three properties mentioned above, then $S=S+\boldsymbol{R}^{+}$. However, for a complete characterization of the aforementioned type of maximal closed preprime a further notion must be introduced.

2. Real and complex places. By a (real, complex) pair in a complex Banach algebra $A$, we mean a pair $(B, B)$ where $B$ is a closed (real, complex) subalgebra of $A$ containing 1 and $B$ is a maximal ideal in $B$. For a real Banach algebra $A$, a real pair is similarly defined. In this chapter we use the term pair to mean either a real or complex pair.

We introduce an order on like pairs in $A$ by setting $\left(B_{1}, B_{1}\right) \leqq\left(B_{2}, B_{2}\right)$ if $B_{1}$ is a closed subalgebra of $B_{2}$ and $\boldsymbol{B}_{2} \cap B_{1}=\boldsymbol{B}_{1}$. A direct application of Zorn's lemma shows that any pair is majorized by a maximal pair. A maximal complex pair is called a complex place. A maximal real pair is called a real place. In the commutative case we also use the terms complex place and real place to denote the corresponding homomorphism from $B$ onto $B / \boldsymbol{B}$. Note that the usage permits, via the GelfandMazur theorem [13, p. 40], a real place to be a homomorphism onto the complexes as well as the reals. The first objective is to relate the notions of a real or complex place and an archimedean maximal closed preprime.

Proposition 2.1. Let $A$ be a real or complex commutative Banach algebra with identity. Then for any place of the form $(A, A), \boldsymbol{A}+\boldsymbol{R}^{+}$is a closed prime.

Proof. The result follows immediately from the Gelfand-Mazur theorem and Example 1.3.

The isolation of the result of Proposition 2.1 is for emphasis. Actually it is a special case of the next proposition. 
Proposition 2.2. Let $A$ be a real or complex commutative Banach algebra with identity. For any place $(B, B)$ of $A, B+\boldsymbol{R}^{+}$is a maximal closed preprime of $A$ which is archimedean.

Proof. Let $S=\boldsymbol{B}+\boldsymbol{R}^{+}$, so that $S$ is a closed archimedean preprime. Suppose that $T$ is a closed preprime and $T \supset S$. Let $D=B T$, so that $D$ is a normed algebra over the same field as $B$, and let $D_{0}=\boldsymbol{B} T \subset T$. Consequently $D_{0}^{-}$is a proper ideal in $D^{-}$and is thus contained in a maximal ideal $\boldsymbol{D}$ of $D^{-}$. Hence, $D^{-} \supset B \supset \boldsymbol{D} \cap B$ $\supset \boldsymbol{B}$. Since $1 \notin \boldsymbol{D}, \boldsymbol{D} \cap B=\boldsymbol{B}$. The maximality of the pair $(B, \boldsymbol{B})$ then implies $B=D^{-} \supset T \supset S$. However, by Proposition $2.1 S$ is a prime in $B$, so $S=T$.

It should be remarked that a place need not be associated with a closed prime or a homomorphism of the original algebra. Let $T$ be the circle and $A=C(T)$. The pair consisting of $B$, the disc algebra, and $\boldsymbol{B}$, the subset of $B$ consisting of the functions whose analytic extension to the disc vanishes at the origin, is easily verified to be a complex place not coming from a homomorphism of $A$. Let $S$ be the associated maximal closed preprime. If $f \in A$ satisfies $f\left(e^{i \theta}\right)=\frac{1}{2}+e^{i \theta}$, then $f \in S^{+}$and there exists $g \in A$ such that $f g=1$. If $S$ were a prime then Proposition 1.6 of [5] yields $g \in S$. But then the extensions $\hat{f}$ and $\hat{g}$ to the disc satisfy $\hat{f} \hat{g}=1$, which is impossible since $f$ has a zero in the interior of the disc. Thus the complex place $(B, \boldsymbol{B})$ is not associated with a prime. It should also be remarked that the above shows that Proposition 1.6 of [5] for primes is false for maximal closed preprimes.

We adopt a notation analogous to that used by Harrison [5]. For $S$ a closed preprime in $A$ we let $A_{S}=\{y \in A \mid y \boldsymbol{S} \subset \boldsymbol{S}$ and $\boldsymbol{S} y \subset \boldsymbol{S}\}$.

Proposition 2.3. Let $A$ be a Banach algebra with identity. Let $S$ be a maximal closed preprime in $A$ which is archimedean and contains $\boldsymbol{R}^{+}$. Then $\boldsymbol{S}=\boldsymbol{S}+\boldsymbol{R}^{+}$. Moreover, $A_{S} / \boldsymbol{S}$ is one of $\boldsymbol{R}, \boldsymbol{C}$, or the quaternions.

Proof. $S$ is a proper closed 2 sided ideal in the real Banach algebra $A_{S}$. Let $A_{0}=A_{S} / S, S_{0}=S / S$, and $B_{0}=S_{0}-S_{0}$. Then $S_{0}$ is both a maximal closed preprime and a cone in $A_{0}$ and for $s_{1}, s_{2} \in S_{0}$, there is an $n \in N$ such that $n-s_{1} \in S_{0}$ and so $n-\left(s_{1}-s_{2}\right) \in S_{0}$. Thus $S_{0}$ defines an order on $B_{0}$ which makes $B_{0}$ into an archimedean ordered algebra in the sense of Kadison [8, Definition 3.1]. Thus by [8, Theorem 3.1] there is an order preserving algebra isomorphism of $B_{0}$ onto a dense subset of $C_{R}(\Omega)$, the algebra of continuous real valued functions on a nonvoid compact set $\Omega$. This isomorphism shows that squares from $B_{0}$ are in $S_{0}$, and guarantees the existence of positive multiplicative functionals on $B$. Let $\varphi$ be any such functional on $B_{0}$. For $y \in B_{0},\|y\| \leqq 1$, let $u=\sum_{i>1} C_{2 i} y^{2 i}$ and $v=\sum_{i>1} C_{2 i+1} y^{2 i}$, where the $C_{i}$ are coefficients in the binomial expansion $(1-t)^{1 / 2}=1-\sum_{i>1} C_{i} t^{i}$. Since each $C_{i} \geqq 0, u, v \in S_{0}$ and consequently $1-u-y v \in B_{0}$ and $1-y \in S_{0}$. Hence $y \in B_{0}$, and $\|y\| \leqq 1$ imply $\varphi(y) \leqq 1$. The use of $-y$ as well as $y$ shows that $\varphi$ is bounded. Extend $\varphi$ to $\left(B_{0}\right)^{-}$by continuity. Then $\varphi^{-1}\left(\boldsymbol{R}^{+}\right)$is a closed preprime in 
$A_{0}$ containing $S_{0}$ so $S_{0}=\varphi^{-1}\left(\boldsymbol{R}^{+}\right)$. This implies that $S_{0}=\left(S_{0} \cap\left(-S_{0}\right)\right)+\boldsymbol{R}^{+}$and that $S=S+\boldsymbol{R}^{+}$.

The final statement of the theorem is a consequence of the Gelfand-Mazur theorem. For if $M$ is a one-sided ideal of $A_{S}$ such that $M \supset S$, then $M^{-}+R^{+}$is a closed preprime in $A_{S}$ containing $S$, so $S=M^{-}+R^{+}$and consequently $A_{S} / S$ is a normed division algebra.

We next show it is possible in Proposition 2.3 that $A$ is an algebra over the complex field, but that $A_{S}$ is not an algebra over $C$. In fact this may even be the case if $A$ is a $C(X)$.

EXAMPLE 2.4. Let $X$ be the closed upper half of the unit circle in $C$, i.e. $X=$ $\left\{e^{i t} \mid 0 \leqq t \leqq \pi\right\}$, and let $A=C(X)$, the complex continuous functions on $X$ with the usual supremum norm. Let $z \in A$ be the usual $z\left(e^{i t}\right)=e^{i t}$. Let

$$
S_{0}=\left\{\alpha_{1} z+\alpha_{2} z^{2}+\cdots+\alpha_{n} z^{n} \mid n \in N \text {, and } \alpha_{j} \in R, j=1, \ldots, n\right\} .
$$

Since each $f \in S_{0}$ is naturally defined on $C$ and $f\left(u^{c}\right)=(f(u))^{c}$, where $c$ denotes complex conjugation, $\sup \{|f(u)+1|: u \in X\}=\sup \{|f(u)+1|: u \in T\}$ with $T$ the unit circle in $C$. For $f \in S_{0}$ the maximum modulus principle yields $1=f(0)+1$ $\leqq \sup \{|f(u)+1|: u \in T\}=\sup \{|f(u)+1|: u \in X\}=\|f+1\|$. Thus $S_{0}^{-}$is a closed preprime and consequently $S_{0}^{-}+\boldsymbol{R}^{+}$is a closed preprime in $A$. Clearly $S_{0}^{-}+\boldsymbol{R}^{+}$ is archimedean and contains $R^{+}$. The pair $\left(S_{0}^{-}+R, S_{0}^{-}\right)$is then majorized by a real place $(B, \boldsymbol{B})$, so by Proposition $2.2 S=\boldsymbol{B}+\boldsymbol{R}^{+}$is an archimedean maximal closed preprime of $A$. It remains to see that $i S \notin S$.

Let $g$ be defined on $X$ by $g(z)=z$. Thus $g \in S_{0} \cap\left(-S_{0}\right)$, and therefore $g \in B$. Suppose that $i g \in \boldsymbol{B}$. Then as $X$ is the maximal ideal space of $A$ and as the Gelfand representation may be viewed as point evaluation, Proposition 1.4 asserts that there is an $x_{0} \in X$, such that $i g\left(x_{0}\right) \geqq 0$. However, this is not true so we conclude that $i \boldsymbol{S} \notin \boldsymbol{S}$.

Proposition 2.5. Every archimedean closed preprime $Q$ which contains $\boldsymbol{R}^{+}$is contained in a maximal closed preprime which is archimedean.

Proof. In the proof of Proposition 2.3, if we start with only an archimedean closed preprime $S$ containing $\boldsymbol{R}^{+}$the argument may be repeated verbatim through the stage where we obtain a homomorphism $\varphi$ with $\varphi^{-1}\left(\boldsymbol{R}^{+}\right)$a closed preprime in $A_{0}$ containing $S_{0}$. If $\pi$ is the natural map of $A_{S}$ onto $A_{0}$, then $\pi^{-1}\left(\varphi^{-1}\left(R^{+}\right)\right)$, is a closed preprime in $A_{S}$ containing $S$. But then $\left(\pi^{-1}\left(\varphi^{-1}(R)\right), \pi^{-1}\left(\varphi^{-1}(0)\right)\right)$ is a real pair and is thus majorized by a real place $(D, D)$. By Proposition $2.2, \boldsymbol{D}+\boldsymbol{R}^{+}$is an archimedean maximal closed preprime and the order on pairs implies $\boldsymbol{D}+\boldsymbol{R}^{+} \supset S$.

Proposition 2.6. Let $A$ be a real or complex commutative Banach algebra. Then any closed prime $P$ is associated with a place $(A, A)$ via the formula $P=A+R^{+}$.

Proof. The results of Propositions 1.6 and 1.10 show that a closed prime $P$ contains $\boldsymbol{R}^{+}$and is archimedean. Thus, since $P$ is also a maximal closed preprime, 
Proposition 2.3 shows that $P=\boldsymbol{B}+\boldsymbol{R}^{+}$with $(B, \boldsymbol{B})$ a place. To complete the proof we must show $B=A$, so we suppose otherwise. For simplicity we consider only the case of $A$ a complex algebra.

Let $x \in A \backslash B$. The expressions of the form $p_{0}+p_{1} x+\cdots+p_{m} x^{m}$, with $p_{0} \in P$, $p_{j} \in \boldsymbol{B}$ for $j=1,2, \ldots, m$ and $m$ some positive integer, are closed under addition and multiplication. The collection contains $P$ properly, since if $\boldsymbol{B} x \subset P$, then $\boldsymbol{B} x \subset \boldsymbol{B}$ and thus $x \in A_{P}=B$ by Proposition 2.3, in contradiction to the choice of $x$. Thus as $P$ is a prime and contains $\boldsymbol{R}^{+}$, there is a polynomial equation of minimal degree $n$ with $-1=p_{0}+p_{1} x+\cdots+p_{n} x^{n}$, and with $p_{0} \in P, p_{j} \in \boldsymbol{B}, j=1, \ldots, n$. Hence

$$
0=p_{n}^{n-1}\left(1+p_{0}\right)+p_{n}^{n-2}\left(p_{n} x\right)+\cdots+\left(p_{n} x\right)^{n} .
$$

Let $I$ be the integral closure of $B$ in $A[15$, p. 256]. Suppose first that $I=B$. The prior equation then shows that $p_{n} x \in I=B$, so $p_{n} x=q_{n}+\lambda$ with $q_{n} \in \boldsymbol{B}$ and $\lambda \in \boldsymbol{C}$. Since Proposition 1.11 asserts that $\boldsymbol{C B}=\boldsymbol{B}$, we may assume $p_{n} x=q_{n}$ or $p_{n} x=q_{n}+1$. If the first formula were to hold, the equation obtained above shows that the integer $n$ is not minimal. Thus $p_{n} x=q_{n}+1$.

As the group $G$ of regular elements of $A$ is open, and we are supposing that $B$ is a proper closed subspace of $A$, the set $G \backslash B \neq \varnothing$ and is open. Moreover, as inversion is continuous where defined, $(G \backslash B)^{-1}$ is also open and hence cannot be in $B$. We may therefore choose $y \in A \backslash B$ such that $y^{-1} \in A \backslash B$. The prior discussion applied to $y^{-1}$ yields $p \in \boldsymbol{B}, q \in \boldsymbol{B}$ and $p y^{-1}=q+1$ or $p=(q+1) y$. Since $q+1 \in P^{+}$, and $(q+1) P y=p P \subset P$, Proposition 1.6 of [5] yields $y \in P \subset B$. This contradiction shows that $I \neq B$.

Since $B$ is a maximal ideal of $B$, it is a prime ideal of $B$. Theorem 3 of $[15$, p. 257] then gives a prime ideal $J$ of $I$ such that $J \cap B=B$. As noted earlier in the argument $A_{P}=B$. Thus Proposition 2.5 of [5] shows that $(B, B)$ is a valuation pair in the sense of Manis [10] (or [11, p. 18]). This asserts that $(B, B)$ is maximal with respect to the order $\left(R_{1}, P_{1}\right) \leqq\left(R_{2}, P_{2}\right)$ if $R_{1}$ and $R_{2}$ are subrings of an overring $R$, in this case $A, P_{j}$ is a prime ideal of $R_{j}, j=1,2, R_{1} \subset R_{2}$ and $P_{2} \cap R_{1}=P_{1}$. However, in the present situation we have $(B, B) \leqq(I, J)$ and $I \neq B$. This contradiction shows that $B=A$.

3. Finite maximal closed preprimes. In this section we examine the behavior of finite maximal closed preprimes in Banach algebras. It is of no consequence whether the algebras are real or complex, and commutativity plays no role in the arguments. The principal result of Theorem 3.4 is that finite maximal closed preprimes automatically introduce other fields as quotients than the traditional reals, complexes and quaternions.

LEMma 3.1. Let $S$ be a maximal closed preprime in the Banach algebra $B$. Then $S$ is a finite maximal closed preprime if and only if $S=-S$. 
Proof. The result in one direction is immediate from the definition of a finite preprime. Suppose then that $S$ is a finite maximal closed preprime and let $N$ denote the nonnegative integers. Then the set $S+N$ is closed under addition and multiplication and properly contains $S$. Thus $-1=\lim _{k \rightarrow \infty}\left(s_{k}+n_{k}\right)$ with each $s_{k} \in S$ and $n_{k} \in N$. For arbitrary $s \in S,-s=\lim _{k \rightarrow \infty}\left(s s_{k}+n_{k} s\right) \in S$ as $S$ is closed.

LEMMA 3.2. Let $S$ be a closed preprime in a Banach algebra $B$, with $S=-S$. Then there is a finite maximal closed preprime $T$ such that $T \supset S$.

Proof. Suppose that the lemma is false. We know that $S$ is contained in some maximal closed preprime $M$, and our supposition implies that only infinite maximal closed preprimes can contain $S$. Let $p$ be any prime number. The set $(S+Z p)^{-}$ is closed under multiplication and addition. If it were a preprime, it would be contained in a maximal closed preprime, which by our supposition must contain 1 as well as $-p$ and we would have a contradiction as $-1=-p+(p-1) 1$. Thus $-1 \in(S+Z p)^{-}$. Let $s_{k} \in S$ and $m_{k} \in Z$ be such that $-1=\lim _{k \rightarrow \infty}\left(s_{k}+m_{k} p\right)$. We note that $m_{k}<0$ for $k \geqq k_{0}$, since otherwise $s_{k}+m_{k} p \in M$ and $M$ is closed. However, for $k \geqq k_{0}$, we have $-m_{k} p-2 \geqq 0$ and since $S=-S,-s_{k}-m_{k} p-2 \in M$ and $-1=\lim _{k \rightarrow \infty}\left(-s_{k}-m_{k} p-2\right)$, which contradicts the fact that $M$ is a closed preprime.

In order to show that the study of finite maximal closed preprimes should be expected to lead to considerations atypical to the study of Banach algebras, let us consider the simplest conceivable real and complex Banach algebras and observe the nature of their finite maximal closed preprimes.

EXAMPLE 3.3. (1) Any finite maximal closed preprime in $\boldsymbol{R}$ has the form $Z p$ for some prime number $p$. (2) To any finite maximal closed preprime $S$ in $C$, there corresponds a positive integer $k$ and algebraic numbers $a_{1}, a_{2}, \ldots, a_{k}$ such that $S=Z a_{1} \oplus$ $\cdots \oplus Z a_{k}$. Moreover, there exists a unique $p \in N \cap S$ which is a prime number.

Proof. (1) For any prime number $p, Z p$ is a closed preprime and is thus contained in a maximal closed preprime $S$, which must be finite since $-p \in S$. Thus by Lemma 3.1, $S$ is a closed subgroup of the additive group $\boldsymbol{R}$. One knows from topological group theory that this implies that $S=Z p$.

Suppose next that $S$ is a finite maximal closed preprime in $\boldsymbol{R}$. As $S$ is a closed proper subgroup of $\boldsymbol{R}, S=Z c$ for some $c>0$, and as $c^{2} \in S$, we see that $c \in N$. If $c$ were not a prime, $S$ would be properly contained in a closed preprime $Z r$, with $r$ some factor of $c$.

(2) Let $S$ be a finite maximal closed preprime in $C$. Lemma 3.7 says $S$ is a subgroup of $\boldsymbol{C}$ as an additive group, and $S$ is closed, so $S$ is a compactly generated locally compact abelian group, so by the structure theory for such groups [6, p. 90] $S$ is isomorphic as a topological group to $\boldsymbol{R}^{m} \times \boldsymbol{Z}^{n} \times F$, where $\{m, n\} \subset N$ and $F$ is a compact group. Since $C$ contains no nonzero compact additive subgroups, the term in $F$ may be deleted. Also, since $S$ is a proper subgroup of $C, m=0$ or 1 . If $m=1$, there would exist some $c \in S,|c|=1$, and $t c \in S$ for all $t \in R$. Thus for 
some $0 \neq t \in \boldsymbol{R}$ we must have $t^{2} c^{2}=u c$ with $u \in \boldsymbol{R}$. But the prior relation forces $c$ to be real, which is impossible. Thus $m=0$. We thus conclude that $S$ is isomorphic as a topological group with $Z^{n}$. In particular $S$ is discrete in $C$, and $S=Z a_{1} \oplus Z a_{2}$ $\oplus \cdots \oplus \boldsymbol{Z} a_{n}$. Since $S$ is closed under addition and multiplication, each $a_{j}, j=1, \ldots$, $n$, is an algebraic number. Suppose that there is no prime number in $N \cap S$. Then $S+N$ is closed under addition and multiplication, and properly contains $S$. Thus $-1=\lim _{k \rightarrow \infty} s_{k}+n_{k}$ with each $s_{k} \in S$ and $n_{k} \in N$. Let $0 \neq s \in S$. Then $-s=\lim _{k \rightarrow \infty}\left(s s_{k}+n_{k} s\right)$. However, $S$ is discrete. Thus for $k$ sufficiently large $-s=s s_{k}+n_{k} s$. Since $s \neq 0,-1=s_{k}+n_{k}$ and we see that $S$ contains some integer. The least positive integer in $S$ must be a prime number.

It is worthy of note that the examples of finite maximal closed preprime in $C$ are necessarily more complicated than those of $\boldsymbol{R}$. For if $p$ is any prime number, then $Z p \oplus Z p^{1 / 2} i$ is a closed finite preprime in $C$, and since Lemma 3.2 is available $Z p \oplus Z p^{1 / 2} i$ is contained in some finite maximal closed preprime.

Before we return to the analysis of the nature of the finite maximal closed preprimes, it is convenient to introduce certain notions which will appear in the sequel. We call a ring $R$ a metric ring (see [9]) if there is associated with each $r \in R$ a real number $|r|$ such that $|r|=0$ if and only if $r=0,|-r|=|r|,|r+s| \leqq|r|+|s|$ and $|r s| \leqq|r||s|$. If in the topology that the norm gives $R, R$ is complete we call $R$ a Banach ring.

Banach rings arise naturally in the study of Banach algebra if one has a closed additive subgroup $S$ of a Banach algebra $B$ with identity, and with $\|1+s\| \geqq 1$ for $s \in S$. Let $D=\{x \in B \mid x S \subset S$ and $S x \subset S\}$, so that $D$ is a closed subring of $B$, and $Z \subset D$, since $n s=s+\cdots+s$, with $n$-fold addition, is in $D$ if $n \in N$. In the quotient ring $D / S$ define $|x+S|=\inf \{\|x+s\|: s \in S\}$, where \|\| is the norm in $B$. That $D / S$ is a metric ring is immediate. The proof of the completeness of $D / S$ is identical with the usual proof for Banach spaces (e.g. [3, p. 26]).

We use the terminology of preprime, closed preprime and so forth for Banach or metric rings exactly as the terms were used for Banach algebras.

Another notion which we encounter is that of locally finite field. This is a field such that each element is contained in a finite subfield. Such fields are known up to isomorphism and any such field is completely characterized by its characteristic and a single additional invariant, a so-called Steinitz number (see [5, p. 9] or [14]). It is these fields which enter into the Harrison theory of primes.

THEOREM 3.4. Let $S$ be a finite maximal closed preprime in a Banach algebra $B$ with identity. Let $B_{S}=\{x \in B \mid x S \subset S$ and $S x \subset S\}$. Then one of the following holds:

(1) If $S \cap N=(0), B_{S} / S$ (as a ring) is a metric ring, any closed subring of which is a division ring and contains as a closed subfield a completion of a copy of the rational numbers distinct from the reals.

(2) If $S \cap N \neq(0), B_{S} / S$ (as a ring) is a locally finite field and is discrete.

Moreover, in either case $B_{S} \subset(R S)^{-}$. 
Proof. The set $B_{S}$ is a closed subring of $B$ and $S$ is an ideal of $B_{S}$. As noted in the introduction of the concept of a Banach ring, $B_{S} / S$ is a Banach ring with identity. Let $\pi$ denote the natural map from $B_{S}$ onto $B_{S} / S$. If $Q$ is a maximal closed preprime in $B_{S} / S$, then $\pi^{-1} Q$ is a closed preprime in $B$ containing $S$ and consequently $Q=(0)$.

We shall show that any nonzero closed subring $D$ of $B_{S} / S$ is a division ring. Let $a \in D, a \neq 0$. Let $C=(D a+Z a)^{-}$. If $-1 \notin C$, then $C$ is a nonzero closed preprime in $B_{S} / S$, contradicting the above. Thus $-1 \in C$ and $1 \in C$. The usual series argument for the existence of an inverse makes no use of scalar multiplication, so is valid here. Suppose $1=\lim \left(d_{n} a+m_{n} a\right), d_{n} \in D, m_{n} \in Z$. Then for sufficiently large $n$, $d_{n} a+m_{n} a$ has an inverse $b_{n}$ in $D$ and consequently $a$ has a left inverse in $D$. An identical argument using $(a D+Z a)^{-}$yields a right inverse for $a$ in $D$.

The proof now diverges into the two possibilities involved in the statement of the theorem. If $S \cap N=(0)$, then $B_{S} / S$ contains $Z+S$, a copy of the integers. If $I$ is the closure in $B_{S} / S$ of the ring $Z+S$ then, as we have seen above, $I$ is a division ring, and as $I$ is clearly commutative, $I$ is a field. Since $I$ contains a copy of the integers as a dense set, it is a completion of a copy of the rational numbers different from the real numbers.

In the second instance, it is readily verified that the smallest positive integer in $S$ is a prime $p$. Consequently $B_{S} / S$ has characteristic $p$. Let $I$ denote the field $Z+S$ in $B_{S} / S$. For simplicity we write the elements of $I$ as $0,1, \ldots, p-1$. Let $|a|$ denote the norm of $a$ in $B_{S} / S$. Suppose $a \in B_{S} / S, a \neq 0$ and $|a|<1$. Let $I[a]$ represent the polynomials in $a$ with coefficients in $I$. Then for $f \in I[a], f=\alpha_{0}+\alpha_{1} a+\cdots+\alpha_{n} a^{n}$, $\alpha_{j} \in I$, one has $|f| \leqq p \sum_{j=0}^{n}\left|a^{j}\right| \leqq p(1-|a|)^{-1}$ since $\left|\alpha_{j}\right| \leqq p$ for all $\alpha \in I$.

Since $|a|<1$, there is an $n \in N$ such that $|a|^{n}<p^{-1}(1-|a|)|1|$. Then as $(I[a])^{-}$ is a field, the comments of the prior paragraph apply to $\left(a^{n}\right)^{-1}$ and $\left|\left(a^{n}\right)^{-1}\right|$ $\leqq p(1-|a|)^{-1}$. Hence, $|1| \leqq\left|a^{n}\right|\left|\left(a^{n}\right)^{-1}\right|<1$, which implies that $B_{S} / S$ is discrete.

Any prime $P$ in $B_{S} / S$ is thus closed. If $\pi$ is the natural map of $B_{S}$ onto $B_{S} / S$, then $\pi^{-1}(P)$ is a closed preprime containing $S$ and hence is $S$. Consequently the only prime in $B_{S} / S$ is (0) and $B_{S} / S$ is a locally finite field by [5, Proposition 1.4].

To see the final assertion, we let $L$ be the smallest closed subring of $B$ containing $B_{S}$ and $1 / 2$. Then $L \supset \boldsymbol{R}$, so is a real Banach algebra. Also $(\boldsymbol{R} S)^{-}$is an ideal of $L$ and contains $S$. Thus if $(R S)^{-}$is a proper ideal of $L$, we would have a contradiction to the fact that $S$ is a maximal closed preprime. Hence, $(R S)^{-}=L \supset B_{S}$.

4. Function algebras. In the present section we consider the nature of infinite maximal closed preprimes in function algebras. By a function algebra we mean a uniformly closed complex algebra $A$ of complex-valued continuous functions on a compact Hausdorff space $X$, such that $A$ contains the constants and separates the points of $X$. There may of course be more than one compact Hausdorff space capable of serving as the domain of the functions in $A$. The largest such domain 
space is the space $\mathfrak{M}(A)$ of maximal ideals, and the smallest is the Shilov boundary $\delta(A)$.

The principal concept of this section is the notion of a minimal $S$-set. Given a function algebra $A$ defined on $X$, an infinite maximal closed preprime $S$ of $A$, and a nonvoid closed subset $Y$ of $X$, we call $Y$ an $S$-set (relative to $X$ ) if $\|f+1\|_{Y} \geqq 1$ for all $f$ in $S$. The sets $X$ and $\delta(A)$ are both $S$-sets. There are two other natural $S$-sets, which we shall mention later. A standard compactness argument shows that any $S$-set contains a minimal $S$-set. The description of the $S$-sets and minimal $S$-sets depends heavily on the domain space $X$, as we will show later by an example.

Let $(B, B)$ be a complex place in the function algebra $A$ defined on $X$. Let $S$ be the corresponding maximal closed preprime, and let $K$ be a minimal $S$-set. We will show that $K$ is a set of antisymmetry for $B$ (see [4]), and that there is a positive Baire measure $m$ on $X$ whose closed support is $K$, and which represents the homomorphism $\varphi$ on $B$ associated with $\boldsymbol{B}$.

If $X$ is a compact Hausdorff space and $A$ is the algebra $C(X)$, then for each infinite maximal closed preprime $S$ of $A$ there is a unique minimal $S$-set, which we denote by $C_{S}$. If we let $J_{S}$ denote the largest ideal of $C(X)$ contained in $S$, then we can assert that $C_{S}$ is the hull of $J_{S}$.

We make a good deal of use in this section of an operation which we call the star product. It was used by Jacobson in evolving the Jacobson radical. For any pair $z_{1}, z_{2}$ of complex numbers, we define $z_{1} * z_{2}=z_{1}+z_{2}+z_{1} z_{2}$. It is well known that $*$ is commutative and associative, and that -1 is its zero element. The set $b(-1,1)=\{z:|z+1| \leqq 1\}$ is a semigroup under $*$, with $\{z:|z+1|<1\}$ as an ideal and $\{z:|z+1|=1\}$ as a subgroup. If $f_{1}$ and $f_{2}$ are in a function algebra $A$, we define $f_{1} * f_{2}=f_{1}+f_{2}+f_{1} f_{2}$.

Many of the results of this section depend on the following lemma.

Lemma 4.1. Let $A$ be a function algebra defined on the compact Hausdorff space $X$, and let $S$ be an infinite maximal closed preprime of $A$. Let $L$ and $M$ be any pair of nonvoid subsets of $X$. Then the following four assertions are true.

(1) If there are functions $f$ and $g$ in $S$ such that $f \equiv-1$ on $L$ and $g \equiv-1$ on $M$, then there is a function $h$ in $S$ such that $h \equiv-1$ on $L \cup M$.

(2) If there are functions $f$ and $g$ in $S$ such that $f \equiv-1$ on $L$ and $\|g+1\|_{M}<1$, then there is a function $h$ in $S$ such that $\|h+1\|_{L \cup M}<1$.

(3) If $\boldsymbol{R}^{+} \subset S$ and if there is a function $f$ in $S$ such that $f^{-1}(0)=L$ and $\|f+1\|=1$, then there is no function $g$ in $S$ such that $\|g+1\|_{L}<1$.

(4) If $\boldsymbol{R}^{+} \subset S$, then for every $f$ in $S$, there is a point $x$ in $X$ such that $f(x) \geqq 0$.

Proof. (1) Let $h=f * g=f+g+f g$.

(2) Let $g_{n}=g * g * \cdots * g$, the $n$-fold star product of $g$ with itself. Then $g_{n} \rightarrow-1$ uniformly on $M$, and $f * g_{n} \rightarrow-1$ uniformly on $M$. Choose an integer $m$ such that $\left\|f * g_{m}+1\right\|<1$. Let $h=f * g_{m}$. Then $h \in S$ and $h \equiv-1$ on $L$, so that $\|h+1\|_{L \cup M}<1$.

(3) We may without loss assume that the function $f+1$ peaks on $L$, since we 
can replace $f$ by $\frac{1}{2} f$ if necessary. Suppose there is a $g$ in $S$ such that $\|g+1\|_{L}<1$. Then there is an open set $U$ containing $L$ such that $\|g+1\|_{U}<1$. Now $X \mid U$ cannot be void, and $\|f+1\|_{X \backslash U}<1$. Let $f_{n}=f * f * \cdots * f$, the $n$-fold star product of $f$ with itself. Then $g * f_{n} \rightarrow-1$ uniformly on $X \backslash U$, while $\left\|g * f_{n}+1\right\|_{U}<1$ for every $n$. There is, therefore, an integer $m$ such that $\left\|g * f_{m}+1\right\|<1$. Since $g * f_{m}$ is in $S$, we have reached a contradiction to Proposition 1.1.

(4) The preprime $S$ is a closed preprime of $C(X)$. Since $R^{+} \subset S$, we may apply Proposition 1.4 and reach the desired conclusion.

Definition 4.2. By a triple $(A, X, S)$ we mean a function algebra $A$ defined on a compact Hausdorff space $X$, with $S$ an infinite maximal closed preprime of $A$. By a couple $(U, f)$ of $(A, X, S)$ we mean a nonvoid open subset $U$ of $X$ and a function $f$ in $S$ such that $f \equiv-1$ on $U$. If $A$ and $X$ are given, we will sometimes refer to $(U, f)$ as a couple of $S$. If $x \in X$, we say that $x$ admits the couple $(U, f)$ if $x \in U$ and $(U, f)$ is a couple of $S$.

Definition 4.3. Let $(A, X, S)$ be given. We define the subset $0_{S}$ of $X$ by the rule $0_{S}=\{x \in X: x$ admits no couple of $S\}$.

Proposition 4.4. The set $0_{S}$ is an $S$-set relative to $X$.

Proof. We must show that $0_{S}$ is a nonvoid closed subset of $X$ such that $\|f+1\|_{0_{S}}$ $\geqq 1$ for all $f$ in $S$. Now $0_{S}$ is closed, since its complement is open. Suppose $0_{S}$ is void. Then each point of $X$ admits a couple of $S$, and since $X$ is compact there is a finite collection of couples, say $\left\{\left(U_{1}, f_{1}\right), \ldots,\left(U_{n}, f_{n}\right)\right\}$, such that $X=U_{1} \cup \cdots \cup U_{n}$. Let $f=f_{1} * \cdots * f_{n}$. Then $f \in S$ and $f \equiv-1$ on $X$, a contradiction. To complete the proof we must show that $\|f+1\|_{0_{S}} \geqq 1$ for every $f$ in $S$. Suppose there is a $g$ in $S$ such that $\|g+1\|_{0_{S}}<1$. Then there is an open subset $U$ of $X$ with $U \neq X$ such that $0_{S} \subset U$ and $\|g+1\|_{U}<1$. Now there is an $f$ in $S$ such that $f \equiv-1$ on $X \backslash U$. By part (2) of Lemma 4.1 there is a function $h$ in $S$ with $\|h+1\|_{X}<1$, a contradiction.

Corollary 4.5. Let $Y$ be any closed subset of $X$ disjoint from $0_{S}$. Then $Y$ is not an S-set.

Corollary 4.6. Let $Y$ be any $S$-set. Then the set $Y \cap 0_{S}$ is also an $S$-set.

Proof. The set $Y \cap 0_{S}$ is closed, and it is nonvoid by the previous corollary. Suppose there is a function $g$ in $S$ with $\|g+1\|_{Y \cap 0_{S}}<1$. Then there is an open set $U$ with $Y \cap 0_{S} \subset U$ such that $\|g+1\|_{U}<1$. The closed set $Y \mid U$ is nonvoid, since $Y$ is an $S$-set and $U^{-}$is not. There is an $f$ in $S$ such that $f \equiv-1$ on $Y \backslash U$. Since $f \equiv-1$ on $Y \backslash U$ and $\|g+1\|_{U}<1$, we can apply part (2) of Lemma 4.1 to obtain an $h$ in $S$ such that $\|h+1\|_{Y}<1$, a contradiction.

We have seen that $0_{S}$ is an $S$-set. Another natural $S$-set (relative to $X$ ) is the set $N_{S}$, which we define in the course of the following discussion.

Definition 4.7. Let the triple $(A, X, S)$ be given, and suppose $R^{+} \subset S$. We define the subset $T_{S}$ of $S$ by the rule $T_{S}=\{f \in S:\|f+1\|=1\}$. 
The set $T_{S}$ is the set of all functions in $S$ which are closest to the function -1 . Clearly 0 is in $T_{S}$.

THEOREM 4.8. Let $(A, X, S)$ be given, and suppose $\boldsymbol{R}^{+} \subset S$. Then the following three assertions hold.

(1) The set $T_{S}$ is closed under star products.

(2) The set $T_{S}$ is a closed convex subset of $A$, and 0 is an extreme point of $T_{S}$.

(3) The set $N_{S}=\bigcap\left\{f^{-1}(0): f \in T_{S}\right\}$ is a nonvoid closed subset of $X$.

Proof. (1) Suppose $f$ and $g$ are in $T_{S}$. Then $f * g$ is in $S$, and

$$
1 \leqq\|(f * g)+1\|=\|f+g+f g+1\|=\|(f+1)(g+1)\| \leqq\|f+1\|\|g+1\|=1 .
$$

Thus $f * g$ is in $T_{S}$.

(2) Suppose $f$ and $g$ are in $T_{S}$, and suppose $\alpha \geqq 0, \beta \geqq 0$ with $\alpha+\beta=1$. Then $\alpha f+\beta g$ is in $S$, and

$$
1 \leqq\|\alpha f+\beta g+1\|=\|\alpha(f+1)+\beta(g+1)\| \leqq \alpha\|f+1\|+\beta\|g+1\|=\alpha+\beta=1 .
$$

Thus $\alpha f+\beta g$ is in $T_{S}$. We omit the easy proof $\alpha$ the fact that 0 is an extreme point of $T_{S}$.

(3) Suppose that the closed set $N_{S}$ is void. Then for each point $x$ of $X$ there is a function $f$ in $T_{S}$ and an open neighborhood $U$ of $x$ such that $f$ does not vanish at any point of $U$. Since $X$ is compact there is a finite collection $\left\{U_{1}, \ldots, U_{n}\right\}$ of nonvoid open subsets of $X$, and an associated collection $\left\{f_{1}, \ldots, f_{n}\right\} \subset T_{S}$ such that $X=U_{1} \cup \ldots \cup U_{n}$ and $f_{i}$ does not vanish at any point of $U_{i}$ for $i=1, \ldots, n$. Let the function $f$ be defined by $f=\left(\frac{1}{2} f_{1}\right) * \cdots *\left(\frac{1}{2} f_{n}\right)$. Then $f \in S$, and $\|f+1\|<1$, a contradiction.

COROLlaRY 4.9. The family $\left\{f^{-1}(0): f \in T_{S}\right\}$ is closed under the formation of finite intersections.

Proof. Let $\left\{f_{1}, \ldots, f_{n}\right\} \subset T_{S}$. Then $\left\{\frac{1}{2} f_{1}, \ldots, \frac{1}{2} f_{n}\right\} \subset T_{S}$ since 0 is in $T_{S}$ and $T_{S}$ is convex. Let $f=\left(\frac{1}{2} f_{1}\right) * \cdots *\left(\frac{1}{2} f_{n}\right)$. Then $f \in T_{S}$ and $f^{-1}(0)=f_{1}^{-1}(0) \cap \cdots \cap f_{n}^{-1}(0)$.

Proposition 4.10. Let $(A, X, S)$ be given and suppose $\boldsymbol{R}^{+} \subset S$. Let $Y$ be any closed subset of $X$ disjoint from $N_{S}$. Then $Y$ is not an $S$-set.

Proof. If $Y=\varnothing$, then $Y$ is not an $S$-set. If $Y$ is nonvoid, then the nonvoid open set $X \backslash Y$ contains $N_{S}$. By the compactness of $X$ and Corollary 4.9 there is an $f$ in $T_{S}$ such that $f^{-1}(0) \subset X \backslash Y$. Now $\frac{1}{2} f \in S$ and $\left\|\frac{1}{2} f+1\right\|_{Y}<1$, so $Y$ is no $S$-set.

Proposition 4.11. Let $(A, X, S)$ be given, with $\boldsymbol{R}^{+} \subset S$. Then $N_{S}$ is an $S$-set.

Proof. The set $N_{S}$ is nonvoid and closed. Suppose it is not an $S$-set. Then there is a $g$ in $S$ with $\|g+1\|_{N_{S}}<1$. There is an open set $U$ with $N_{S} \subset U$ and $\|g+1\|_{U}<1$. As in the proof of the previous corollary, there is an $f$ in $T_{S}$ such that $N_{S} \subset f^{-1}(0)$ 
$\subset U$. Let $L=f^{-1}(0)$. Then $\|g+1\|_{L}<1$, and we have reached a contradiction to part (3) of Lemma 4.1.

Corollary 4.12. Let $(A, X, S)$ be given, with $\boldsymbol{R}^{+} \subset S$. Let $Y$ be any $S$-set. Then $Y \cap N_{S}$ is an S-set.

Proof. The set $Y \cap N_{S}$ is a closed subset of $X$, and it is nonvoid by Proposition 4.10. Suppose there is a $g$ in $S$ with $\|g+1\|_{Y_{\cap} N_{S}}<1$. Then $\|g+1\|_{U}<1$ for some open set $U$ containing $Y \cap N_{S}$. The closed set $Y \backslash U$ is nonvoid, since $Y$ is an $S$-set and $U^{-}$is not. There is an $f$ in $T_{S}$ such that $\|f+1\|_{Y \backslash U}<1$ and $f^{-1}(0) \cap Y \subset U$. The argument in the proof of part (3) of Lemma 4.1 shows that $Y$ is not an $S$-set, a contradiction.

Proposition 4.13. Let $(A, X, S)$ be given and let $Y$ be any $S$-set (relative to $X$ ). Then $Y$ contains a minimal $S$-set.

Proof. Let $\left\{Y_{\alpha}: \alpha \in \Lambda\right\}$ be an indexed chain of $S$-sets such that each $Y_{\alpha} \subset Y$. By Zorn's lemma it is sufficient to show that $Y_{0}=\bigcap\left\{Y_{\alpha}: \alpha \in \Lambda\right\}$ is an $S$-set. Suppose it is not an $S$-set. Then for some $f$ in $S,\|f+1\|_{Y_{0}}<1$. There is an open set $U$ with $Y_{0} \subset U$ such that $\|f+1\|_{U}<1$. Since the $Y_{\alpha}$ 's form a chain there is an index $\beta$ such that $Y_{0} \subset Y_{\beta} \subset U$. Thus $\|f+1\|_{Y_{\beta}}<1$, contradicting the fact that $Y_{\beta}$ is an $S$-set.

TheOREM 4.14. Let $(A, X, S)$ be a given triple, and let $K$ be a minimal $S$-set. Then the following assertions hold:

(1) Let $S^{\prime \prime}=S \mid K=\{f \mid K: f \in S\}$. Then $S^{\prime}$, regarded as a preprime of

$$
A \mid K=\{g \mid K: g \in A\},
$$

is such that $0_{S^{\prime}}=K$.

(2) Suppose $\boldsymbol{R}^{+} \subset S$. Then $N_{S^{\prime}}=K$.

(3) $K \subset 0_{s}$.

(4) Suppose $\boldsymbol{R}^{+} \subset S$. Then $K \subset N_{S}$.

(5) Suppose $\boldsymbol{R}^{+} \subset S$. Then $K$ is either a singleton or a perfect set.

(6) Suppose that $S$ is archimedean and contains $R^{+}$. Then $K$ is a set of antisymmetry for the Banach algebra $B$, where $B=\{f \in A: f S \subset S\}$. That is, if $f$ is in $B$ and $f$ is real-valued throughout $K$, then $f$ is constant on $K$. Moreover, to each element in $A \mid K$ there is a unique best approximant in $S \mid K$.

Proof. (1) By definition $0_{S^{\prime}} \subset K$. Moreover $0_{S^{\prime}}$ is an $S^{\prime}$-set, and so an $S$-set. Therefore $0_{S^{\prime}}=K$.

(2) The same argument applies, with $N_{S^{\prime}}$ in place of $0_{S^{\prime}}$.

(3) By Corollary 4.6, $K \cap 0_{S}$ is an $S$-set. Since $K$ is minimal, $K \subset 0_{S}$.

(4) By Corollary 4.12, $K \cap N_{S}$ is an $S$-set. Therefore $K \subset N_{S}$.

(5) Suppose $K$ is not a singleton. We must show that $K$ possesses no isolated point. Suppose that $x$ is an isolated point of $K$. Then $\{x\}$ is relatively open in $K$, 
and so part (1) of this theorem shows that there is no $f$ in $S$ with $f(x)=-1$. Therefore, by Example 1.3, every $f$ in $S$ is such that $f(x) \geqq 0$. Thus $\{x\}$ is an $S$-set which is strictly smaller than $K$, a contradiction.

(6) We first prove the assertion about antisymmetry. By Proposition 2.3, $S=S+R^{+}$. According to that same proposition, either $B=S \oplus R$ or $B=S \oplus C$, depending on whether or not the $\boldsymbol{R}$-module $\boldsymbol{S}$ is in fact a $\boldsymbol{C}$-module. We treat the two cases separately. Suppose first that $B=S \oplus \boldsymbol{R}$. In this case it is sufficient to show that if $f$ is in $S$ and is real-valued throughout $K$, then $f$ vanishes on all of $K$. For such an $f$, the function $-f^{2}$ is in $S$, and so for some $\delta>0,-\delta f^{2} \mid K \in T_{S}$. But $T_{S^{\prime}}=\{0\}$ by part (2) above. Therefore $-\delta f^{2} \equiv 0$ on $K$, so $f \equiv 0$ on $K$. Now suppose that $B=S \oplus C$. If $f \in S$ and $f$ is real-valued on $K$, then $f \equiv 0$ on $K$. Let $f \in B$, and suppose $f$ is real-valued on $K$. Then $f=g+\beta$ where $g \in \boldsymbol{S}$ and $\beta \in \boldsymbol{C}$. Now $\operatorname{Im} \beta=0$, for otherwise the function $g=f-\beta$ never takes on a real value on the set $K$, thus contradicting part (4) of Lemma 4.1, applied to the closed preprime $(S \mid K)^{-}$. Therefore $\beta$ is a real number and $f \equiv \beta$ on $K$.

Finally, if $f \in B$ and $f=g+\beta$ where $g \in S$ and $\beta \in C$, then the fact that $T_{S^{\prime}}=\{0\}$ implies that $g \mid K$ is the unique element of $S \mid K$ which is closest to $f \mid K$.

Proposition 4.15. Let $(A, X, S)$ be a given triple, and let $Y$ be any $S$-set. Then for every $f$ in $A, f$ is in $S$ if and only if $f \mid Y$ is in $S \mid Y$.

Proof. Let $T=\{f \in A: f|Y \in S| Y\}$. Since $f \in T \Rightarrow\|f+1\| \geqq\|f+1\|_{Y} \geqq 1$, we conclude that $T^{-}$is a closed preprime. But $S \subset T \subset T^{-}$, and so $S=T$.

We have concluded our discussion of general function algebras. In the remainder of the section we will discuss some special results on infinite maximal closed preprimes of $C(X)$. The results obtained for function algebras assume a neat form when $A=C(X)$. The structure of closed ideals in $C(X)$ is important in this connection, and so also is the fact that $X$ is (up to homeomorphism) the only compact Hausdorff space which can serve as the domain of the functions in $C(X)$.

Definition 4.16. Let $S$ be an infinite maximal closed preprime in $C(X)$. Let $J_{S}$ be the largest ideal of $C(X)$ contained in $S$, and let $C_{S}=\bigcap\left\{f^{-1}(0): f \in J_{S}\right\}$.

We note that since $S$ is closed, $J_{S}$ is closed. Consequently

$$
J_{S}=\left\{f \in C(X): f \equiv 0 \text { on } C_{S}\right\} .
$$

Proposition 4.17. Let $S$ be an infinite maximal closed preprime of $C(X)$. Then $C_{S}$ is an $S$-set.

Proof. Suppose $C_{S}$ is not an $S$-set. Then there is some $g$ in $S$ such that $\|g+1\|_{C_{S}}$ $<1$. There is an open set $U$ such that $C_{S} \subset U$ and $\|g+1\|_{U}<1$. Clearly $U \neq X$, so $X \backslash U$ is a nonvoid closed subset of $X$ disjoint from $C_{S}$. Let $f \in C(X)$ be such that $f \equiv 0$ on $C_{S}$ and $f \equiv-1$ on $X \backslash U$. Then $f \in J_{S} \subset S$. We now have $f \in S$ and $g \in S$ such that $f \equiv-1$ on $X \backslash U$ and $\|g+1\|_{U}<1$. By part (2) of Lemma 4.1 there is an $h$ in $S$ with $\|h+1\|_{X}<1$, a contradiction. 
Proposition 4.18. Let $S$ be an infinite maximal closed preprime of $C(X)$. Then $C_{S}$ is the unique minimal $S$-set.

Proof. Let $Y$ be any $S$-set. We must show that $C_{S} \subset Y$. By Proposition 4.15, $S$ contains the ideal $J$ of all functions in $C(X)$ which vanish on $Y$. Therefore $J \subset J_{S}$ and $C_{S} \subset Y$.

Proposition 4.19. Let $S$ be an infinite maximal closed preprime of $C(X)$. Then $0_{S}=C_{S}$. If $\boldsymbol{R}^{+} \subset S$, then $N_{S}=C_{S}$.

Proof. By the previous proposition, $C_{S} \subset 0_{S}$. If $x \notin C_{S}$, then there is an open neighborhood $U$ of $x$ such that $U^{-} \cap \cap C_{S}=\varnothing$, and an $f$ in $C(X)$ with $f \equiv 0$ on $C_{S}$ and $f \equiv-1$ on $U^{-}$. Then $f \in J_{S} \subset S$ and $x$ admits the couple $(U, f)$. Consequently $x \notin 0_{S}$, and so $C_{S}=0_{S}$. If $\boldsymbol{R}^{+} \subset S$, then $C_{S} \subset N_{S}$. If $x \notin C_{S}$, then there is some $f$ in $C^{R}(X)$ such that $f \equiv 0$ on $C_{S}, f(x)=-1$, and $-1 \leqq f \leqq 0$. Now $f \in T_{S}$ and $f(x) \neq 0$, so $x \notin N_{S}$. Therefore $C_{S}=N_{S}$.

THEOREM 4.20. Let $S$ be a maximal closed preprime of $C(X)$ such that $\boldsymbol{R}^{+} \subset S$ and $S$ is closed under conjugation. Then for some point $x$ in $X, S=\{f \in C(X): f(x) \geqq 0\}$.

Proof. First we will show that $S$ is archimedean. Since $\boldsymbol{R}^{+} \subset S$, it suffices to show that $(1-f) \in S$ whenever $f \in S$ and $\|f\|<1$. Suppose $f \in S$ and $\|f\|<1$. Then $(1-f)^{-1} \in S$, and so $(1-\bar{f})^{-1} \in S$. Suppose $(1-f) \notin S$. Then there is a finite collection $\left\{f_{0}, f_{1}, \ldots, f_{n}\right\} \subset S$ such that $\left\|1+f_{0}+f_{1}(1-f)+\cdots+f_{n}(1-f)^{n}\right\|<1$. Let $h=f_{0}+f_{1}(1-f)+\cdots+f_{n}(1-f)^{n}$. Then $\operatorname{Re} h<0$ on $X$. Let $g=(1-f)^{-n}(1-\bar{f})^{-n}$. Then $g \in S$ and $g>0$ on $X$. Let $k=g h$. Then $k \in S$ and $\operatorname{Re} k<0$ on $X$, contradicting part (4) of Lemma 4.1.

Next we will show that there is a point $x$ in $X$ such that $f(x)=0$ for all $f$ in $S$. Suppose there is no such point. Then for each point $x$ in $X$ there is a function $f$ in $S$ and an open neighborhood $U$ of $x$ such that $f(y) \neq 0$ for all $y$ in $U$. Since $X$ is compact there is a finite collection $\left\{U_{1}, \ldots, U_{n}\right\}$ of nonvoid open sets and an associated collection $\left\{f_{1}, \ldots, f_{n}\right\} \subset S$ such that $X=U_{1} \cup \cdots \cup U_{n}$ and $f_{i}(y) \neq 0$ for all $y$ in $U_{i}, i=1, \ldots, n$. Now $f \in S$, where $f=f_{1}\left(-\bar{f}_{1}\right)+\cdots+f_{n}\left(-\bar{f}_{n}\right)=-\sum_{i=1}^{n}\left|f_{i}\right|^{2}$. Now $f<0$ on $X$; contradicting part (4) of Lemma 4.1. Therefore there is a point $x$ in $X$ such that $f(x)=0$ for all $f$ in $S$.

By Proposition $2.3 S=S+\boldsymbol{R}^{+}$. Therefore $S=\{f \in C(X): f(x) \geqq 0\}$.

COROLlaRY 4.21. Let $S$ be a maximal closed preprime of $C^{R}(X)$, and suppose that $\boldsymbol{R}^{+} \subset S$. Then there is a point $x$ in $X$ such that $S=\left\{f \in C^{R}(X): f(x) \geqq 0\right\}$.

Proof. The corollary can be proved in the same manner as the theorem. The proof of the corollary is simpler, since conjugation plays no role.

By the last corollary we see that any maximal closed preprime $S$ of $C^{R}(X)$ which contains $\boldsymbol{R}^{+}$is archimedean. However, we do not know whether or not a maximal closed preprime in $C(X)$ which contains $\boldsymbol{R}^{+}$is necessarily archimedean. 
If it is true that $\boldsymbol{R}^{+} \subset S$ implies that $S$ is archimedean for every maximal closed preprime $S$ of $C(X)$ and every compact Hausdorff space $X$, then the same implication holds for an arbitrary function algebra. For if the triple $(A, X, S)$ is given, with $R^{+} \subset S$, then $S \subset S_{1}$ for some maximal closed preprime $S_{1}$ of $C(X)$. If $S_{1}$ is archimedean, then so is $S$, since $S=S_{1} \cap A$.

The next result, which is similar to the first assertion in Theorem $4.14(6)$, is all we can say at present for the maximal closed preprimes of $C(X)$ which contain $\boldsymbol{R}^{+}$.

Proposition 4.22. Let $S$ be a maximal closed preprime of $C(X)$ which contains $\boldsymbol{R}^{+}$. Then $C_{S}$ is a set of antisymmetry for $S$.

Proof. Since $S \mid C_{S}$ is a maximal closed preprime of $C\left(C_{S}\right)$, there is no loss in assuming that $C_{S}=X$. Let $S^{R}=S \cap C^{R}(X)$. We must show that every function in $S^{R}$ is constant.

Our first goal is to show that $S^{R}$ is a closed type 0 semialgebra in the sense of Brown [2]. Surely $S^{R}$ is a closed semialgebra containing $\boldsymbol{R}^{+}$. We must show that $(1+f)^{-1} \in S$ whenever $f \in S^{R}$ and $(1+f)^{-1}$ exists in $C^{R}(X)$. If $f$ is as above then it is a straightforward matter to show that the strictly positive function $(1+f)^{-2}$ is in $S$. But now $(1+f)^{-1}=(1+f)(1+f)^{-2} \in S$. Thus $S^{R}$ is a closed relatively type 0 semialgebra.

It is now an easy consequence of the main representation theorem of [2] that $S^{R}$ is closed under the usual lattice operations for $C^{R}(X)$. We now claim that $f \geqq 0$ for every $f$ in $S^{R}$. For given $f$ in $S^{R}, f \wedge 0$ is in $S^{R}$, so $\delta(f \wedge 0) \in T_{S}$ for some $\delta>0$. Now $C_{S}=X$, so $T_{S}=\{0\}$, and so $f \geqq 0$.

Now suppose $f \in S^{R}$ and $f$ is not constant. Then there are nonnegative reals $\alpha, \beta$, and $\gamma$ such that $\inf f=\alpha<\beta<\gamma=\sup f$. Consider the function $f_{\beta}=f \wedge \beta$. Then $f_{\beta} \in S^{R}$ and $f_{\beta}$ takes on the distinct values $\alpha$ and $\beta$. Let $U=f^{-1}((\beta, \infty))$. Then $U$ is a nonvoid proper open set and $f_{\beta} \equiv \beta$ on $U$. Now the nonnegative function $\beta-f_{\beta}$ is in $S^{R}$ since $S^{R}$ is a closed relatively type 0 semialgebra. Now $\beta-f_{\beta} \equiv 0$ on $U$, and since the nonvoid closed set $X \backslash U$ is no $S$-set, there is a sequence $\left\{g_{n}\right\} \subset S$ with $g_{n} \rightarrow-1$ uniformly on $X \backslash U$. Therefore $g_{n}\left(\beta-f_{\beta}\right) \rightarrow f_{\beta}-\beta$ uniformly on $X$, so $f_{\beta}-\beta \in S$. Now $f_{\beta}-\beta \leqq 0$ and $f_{\beta}-\beta \geqq 0$. Therefore $f_{\beta} \equiv \beta$ on $X$, contradicting the fact that $f_{\beta}$ takes on the distinct values $\alpha$ and $\beta$.

Since the notions of $T$-set and minimal $T$-set can be defined for any closed preprime $T$, we have the following two corollaries.

Corollary 4.23. Let $T$ be a closed preprime of $C(X)$ with $R^{+} \subset T$, and let $K$ be any minimal $T$-set. Then $K$ is a set of antisymmetry for $T$.

Proof. Let $J$ be the ideal of all functions in $C(X)$ which vanish on all of $K$. Then the closed preprime $(T+J)^{-}$is contained in a maximal closed preprime $S$, and $K=C_{S}$.

COROLlARY 4.24. Let $T$ be a closed preprime of $C^{R}(X)$ such that $R^{+} \subset T$. Then any minimal $T$-set $K$ is a singleton. 
Note that Corollary 4.24 implies Corollary 4.21.

THeOrem 4.25. Let $X$ be the finite discrete space $\left\{x_{1}, \ldots, x_{n}\right\}$. Let $S$ be an infinite maximal closed preprime of $C(X)$. Then $S=\{f \in C(X): f(x) \geqq 0\}$ for some $x$ in $X$.

Proof. First we will show that if $\boldsymbol{R}^{+} \subset S$, then $S$ has the desired form. Then we will show that $S$ necessarily contains $\boldsymbol{R}^{+}$. Suppose that $\boldsymbol{R}^{+} \subset S$. Then by part (5) of Theorem 4.14 the set $C_{S}$ is a singleton, so $S$ has the desired form.

Next we will show that $S$ must contain $\boldsymbol{R}^{+}$. Suppose $\boldsymbol{R}^{+}$is not contained in $S$. Then $\frac{1}{2} \notin S$. Therefore there is a finite subset of $S$, say $\left\{f_{0}, f_{1}, \ldots, f_{n}\right\}$ such that $\left\|1+f_{0}+f_{1}\left(\frac{1}{2}\right)+\cdots+f_{n}\left(\frac{1}{2}\right)^{n}\right\|<1$. Therefore $\operatorname{Re}(f)<0$ and $f \in S$, where $f=2^{n} f_{0}$ $+2^{n-1} f_{1}+\cdots+f_{n}$. Let $z_{j}=f\left(x_{j}\right)=a_{j}+i b_{j}$ with $a_{j}$ and $b_{j}$ real, for $j=1, \ldots, n$. Then $a_{j}<0$ for $j=1, \ldots, n$. Now the polynomial $\left(z-z_{j}\right) \cdot\left(z-\bar{z}_{j}\right)$ is a polynomial in $z$ with real positive coefficients, so the same is true of the polynomial $Q$, where $Q(z)=a_{2 n} \prod_{j=1}^{n}\left(z-z_{j}\right)\left(z-\bar{z}_{j}\right)$ and $a_{2 n}$ is a positive integer, as yet undetermined. We see that $Q(z)=\sum_{j=0}^{2 n} a_{2 n} \delta_{j} z^{j}$, where $\delta_{2 n}=1$ and the remaining $\delta_{j}$ are the elementary symmetric functions of the numbers $\left\{z_{j}, \bar{z}_{j}: j=1, \ldots, n\right\}$. Let $P(z)=\sum_{j=0}^{2 n} a_{j} z^{j}$ where the $a_{j}$ are undetermined positive integers. Since $Q\left(z_{j}\right)=0$ for $j=1, \ldots, n$, we know that $\left|P\left(z_{j}\right)\right|=\left|P\left(z_{j}\right)-Q\left(z_{j}\right)\right|, j=1, \ldots, n$. Therefore $\left|P\left(z_{j}\right)\right| \leqq \sum_{i=0}^{2 n}\left|a_{2 n} \delta_{i}-a_{i}\right|$ $\cdot\left|z_{j}\right|^{i}$. By a result in Niven [11, p. 47] we know that for any $\varepsilon>0$ there is a finite set of integers, say $\left\{a_{0}, a_{1}, \ldots, a_{2 n}\right\}$ such that $\left|a_{2 n} \delta_{j}-a_{j}\right|<\varepsilon$ for $j=0,1, \ldots, 2 n-1$. We may without loss assume that $a_{2 n}>0$. If we also require that

$$
\varepsilon<\min \left\{\delta_{0}, \delta_{1}, \ldots, \delta_{2 n-1}, 1\right\},
$$

then each $a_{j}$ is a positive integer. We have shown that it is possible to find a polynomial $P$ with positive integral coefficients, including a positive integral constant term, such that $\left|P\left(z_{j}\right)\right|<1$ for $j=1, \ldots, n$. Then $P \circ f \in S$ and $\|(P \circ f)\|<1$. Let $g=(P \circ f)-1$. Then $g \in S$ and $\|g+1\|=\|P \circ f\|<1$, a contradiction. Therefore $\boldsymbol{R}^{+} \subset S$, so $S$ has the desired form.

Corollary 4.26. Let $X=\left\{x_{1}, \ldots, x_{n}\right\}$ and let $S$ be an infinite maximal closed preprime of $C^{R}(X)$. Then $S=\left\{f \in C^{R}(X): f(x) \geqq 0\right\}$ for some $x$ in $X$.

We close the paper by pointing out a way in which the methodology of measure theory may be brought to bear on maximal closed preprimes which are associated with complex places.

THEOREM 4.27. Let $S$ be a maximal closed preprime of $C(X)$ which is associated with a complex place $\varphi$. Then there is a positive Baire measure $m$ on $X$ such that $C_{S}$ is the closed support of $m$ and $\int f d m=\varphi(f)$ for all $f$ in $B$, where $B=S \oplus C$.

Proof. By a result of Glicksberg [4, p. 419], there is a positive Baire measure $m$ on $X$ of total mass 1 such that $m$ represents $\varphi$ and the closed support of $m$ is a set of antisymmetry for the algebra $B$. Let $K$ be the closed support of $m$. Then $K$ is an 
$S$-set, so $C_{S} \subset K$. Since no strict superset of $C_{S}$ can be a set of antisymmetry for $B$, we conclude that $C_{S}=K$.

THEOREM 4.28. Let $(A, X, S)$ be a given triple and suppose that $S$ is associated with a complex place $\varphi$ of $A$. Let $K$ be any minimal $S$-set relative to $X$. Then there is $a$ positive Baire measure $m$ on $X$ whose closed support is $K$, such that $m$ represents $\varphi$ on $B$, where $B=S \oplus C$.

Proof. By the previous theorem it is sufficient to show that there is a maximal closed preprime $S_{1}$ of $C(X)$ such that $S \subset S_{1}, C_{S_{1}}=K$, and $S_{1}$ is associated with a complex place of $C(X)$. Since $K$ is an $S$-set, $(S \mid K)^{-}$is a closed preprime of $C(K)$. First we will show that $(S \mid K)^{-}=(S \mid K)^{-}+\boldsymbol{R}^{+}$. Suppose $g \in(S \mid K)^{-}$. Then there is a sequence $\left\{g_{n}\right\} \subset S$ such that $g_{n} \rightarrow g$ uniformly on $K$. Therefore the set of numbers $\left\{\left\|g_{n} \mid K\right\|: n \in N\right\}$ is bounded. Each $g_{n}$ can be written in the form $g_{n}=f_{n}+\alpha_{n}$, where $f_{n} \in \boldsymbol{S}$ and $\alpha_{n} \in \boldsymbol{R}^{+}$. Now the sequence $\left\{\alpha_{n}\right\}$ is also bounded. For if $\left\{\alpha_{n}\right\}$ is not bounded then there is a subsequence, still called $\left\{\alpha_{n}\right\}$, such that $\alpha_{n} \rightarrow \infty$. We assume each $\alpha_{n}>0$. Since $\left\{\left\|g_{n} \mid K\right\|: n \in N\right\}$ is bounded, $\alpha_{n}^{-1} g_{n}=\alpha_{n}^{-1}\left(f_{n}+\alpha_{n}\right) \rightarrow 0$ uniformly on $K$. Therefore, for $n$ sufficiently large, we see that $\left\|\alpha_{n}^{-1} f_{n}+1\right\|_{K}<1$, contradicting the fact that $K$ is an $S$-set. Therefore $\left\{\alpha_{n}\right\}$ is a bounded sequence in $\boldsymbol{R}^{+}$, so there is a subsequence $\left\{\alpha_{n_{k}}\right\}$ and a number $\alpha_{0} \in \boldsymbol{R}^{+}$such that $\alpha_{n_{k}} \rightarrow \alpha_{0}$. Therefore $g=\left(g-\alpha_{0}\right)+\alpha_{0}$ is the desired representation of $g$ as a member of $(\boldsymbol{S} \mid K)^{-}+\boldsymbol{R}^{+}$.

Now $\left((S \mid K)^{-} \oplus C,(S \mid K)^{-}\right)$is a complex pair of $C(K)$, and so is majorized by a complex place $(B, \boldsymbol{B})$ of $C(K)$, and $\boldsymbol{B}+\boldsymbol{R}^{+}$is a maximal closed preprime of $C(K)$. Let $T=\boldsymbol{B}+\boldsymbol{R}^{+}$. Let $S_{1}=\{f \in C(X): f|K \in T| K\}$. It is easy to check that $S_{1}$ is a maximal closed preprime of $C(X)$ such that $R^{+} \subset S_{1}, S_{1}$ is archimedean, $S_{1}$ is a $C$-module, and $C_{S_{1}}=K$. By Proposition 2.3 $S_{1}$ is associated with a complex place of $C(X)$, and the present theorem now follows.

EXAmple 4.29. Let $A=C(T)$, and let $(B, B)$ be the complex place of $A$ given by the disc algebra $B$ together with the maximal ideal $B$ of all functions in $B$ which vanish at the origin. Let $S=B+R^{+}$. Then $S$ is a maximal closed preprime of $A$. Since (0) is the largest ideal of $A$ contained in $B, C_{S}=T$, and $T$ is the only $S$-set. However, $S$ is a closed prime in $B$, and $(B, X, S)$ is a triple, where $X$ is the closed unit disc. There are uncountably many minimal $S$-sets relative to $X$. Two of these are $T$ and $\{0\}$. For $0<r<1$ let $K_{r}$ denote the circle of radius $r$ and center 0 . Each $K_{r}$ is an $S$-set by the maximum modulus principle. Actually, each $K_{r}$ is a minimal $S$-set, by part (4) of Lemma 4.1 and the fact that $S$ is a $C$-module containing the function $g$, where $g(z)=z$.

\section{REFERENCES}

1. N. Bourbaki, Eléments de mathématiques. Algèbre commutative. Fasc. 27, Chapitre 1: Modules plats; Chapitre 2: Localisation, Actualités Sci. Indust., no. 1290, Hermann, Paris, 1961. MR 36 \#146. 
2. G. Brown, Relatively type 0 semi-algebras, Quart. J. Math. Oxford Ser. (2) 18(1967), 289-291. MR 36 \#1983.

3. M. M. Day, Normed linear spaces, 2nd rev. ed., Ergebnisse der Mathematik und ihrer Grenzgebiete, Heft 21, Academic Press and Springer-Verlag, New York, 1962. MR 26 \#2847.

4. Irving Glicksberg, Measures orthogonal to algebras and sets of antisymmetry, Trans. Amer. Math. Soc. 105 (1962), 415-435. MR 30 \#4164.

5. D. K. Harrison, Finite and' infinite primes for rings and fields, Mem. Amer. Math. Soc. No. 68 (1966). MR 34 \#7550.

6. E. Hewitt and K. A. Ross, Abstract harmonic analysis. Vol. I: Structure of topological groups. Integration theory, group representations, Academic Press, New York and SpringerVerlag, Berlin, 1963. MR 28 \#158.

7. E. Hewitt and H. S. Zuckerman, Approximation by polynomials with integral coefficients, a reformulation of the Stone-Weierstrass theorem, Duke Math. J. 26 (1959), 305-324. MR 26 \#6656.

8. R. V. Kadison, A representation theory for commutative topological algebra, Mem. Amer. Math. Soc. No. 7 (1951). MR 13, 360.

9. I. Kaplansky, Topological rings, Amer. J. Math. 69 (1947), 153-183. MR 8, 434.

10. M. E. Manis, Extension of valuation theory, Bull. Amer. Math. Soc. 73 (1967), 735-736. MR 36 \#1436.

11. _ Valuations on a commutative ring, Ph.D. Thesis, University of Oregon, Eugene, Oregon, 1966.

12. I. M. Niven, Irrational numbers, Carus Mathematical Monographs, no. 11, The Mathematical Association of America, Wiley, New York, 1956. MR 18, 195.

13. C. E. Rickart, General theory of Banach algebras, The University Series in Higher Mathematics, Van Nostrand, Princeton, N. J., 1960. MR 22 \#5903.

14. E. Steinitz, Algebraische Theorie der Körper, Chelsea, New York, 1950. MR 12, 238.

15. O. Zariski and P. Samuel, Commutative algebra, Vol. I, The University Series in Higher Mathematics, Van Nostrand, Princeton, N. J., 1958. MR 19, 833.

\author{
UNIVERSITY OF OREGON, \\ EUGENE, OREGON \\ UNIVERSITY OF NeW HAMPSHIRE, \\ Durham, New HampshiRe
}

